\title{
Short communication: Statistical models for the analysis of coagulation traits using coagulating and noncoagulating milk information
}

\author{
A. Cecchinato and P. Carnier ${ }^{1}$ \\ Department of Animal Science, University of Padova, viale dell'Università 16, 35020 Legnaro, Padova, Italy
}

\begin{abstract}
Aims of this study were to propose statistical models for the analysis of rennet coagulation time (RCT) suitable for making use of coagulating and noncoagulating (NC) milk information, to estimate heritabilities and to obtain rank correlations for sire merit. A total of 1,025 Holstein cows (progeny of 54 sires) reared in 34 herds were milk-sampled once. Data were analyzed using 4 alternative models: a standard linear (SLM), a right-censored linear Gaussian (CLM), a survival (SUM), and a threshold (THM) model. Model SLM analyzed coagulated milk records only, whereas analysis with CLM or SUM considered information of NC samples as censored records. Model THM analyzed occurrence of milk coagulation as a dichotomous trait. An artificial censoring scenario with an endpoint at 18 min (SET18) was considered after the rearrangement of the timeframe originally used for the observation of RCT (SET31). Heritabilities ranged from 0.12 to 0.25 . Correlations of sire rankings ranged from 0.23 to 0.92 . Differences in sire rankings between SLM and CLM or SUM increased when the proportion of $\mathrm{NC}$ records increased. Correlations between sire rankings obtained for SET31 and SET18 were high for CLM and SUM, indicating that rankings provided by these models tended to be stable even when a large fraction of samples with observed RCT was re-classified as NC milk. Results indicate that CLM and SUM are more suitable than SLM and THM for the analysis of coagulation ability when data contain NC milk information.
\end{abstract}

Key words: milk coagulation time, censoring, statistical model, dairy cattle

\section{Short Communication}

The most frequently used instruments to assess milk coagulation properties (MCP) are milk coagulation meters by which rennet coagulation time (RCT) is measured after addition of the clotting enzyme to raw

Received October 8, 2010.

Accepted April 15, 2011.

${ }^{1}$ Corresponding author: paolo.carnier@unipd.it milk. Time of analysis is usually limited to $31 \mathrm{~min}$, as this is the time at which the curd is cut during the cheese-making process (Ikonen et al., 1999) and milk that does not coagulate within 31 min after rennet addition is considered noncoagulating (NC) milk. This limitation of the timeframe to observe milk coagulation leads to a set of measures with some mathematical peculiarities that may impair further estimates when NC milk information is not properly accounted for in statistical models.

In studies investigating MCP (Ikonen et al., 1999; Cassandro et al., 2008; Vallas et al., 2010), records of NC milk are not used because of missing information on RCT and the inability of the linear model to cope with NC milk records. Alternatively, these records are considered after performing a categorization on the binary scale (Tyrisevä et al., 2004), albeit this approach suffers from a severe information loss. Both approaches involve a rough overcome of data peculiarities: milk samples with very unsatisfactory MCP are omitted from the analysis, biasing the estimation of location and dispersion parameters of RCT, or samples exhibiting different time of curd formation are treated alike and continuous variation of RCT for coagulated samples is neglected.

Because of restrictions in the timeframe to observe coagulation, MCP assessment produces right-censored records when no coagulation occurs. Methods have been proposed to deal with the analysis of censored data. Under a Bayesian framework, Sorensen et al. (1998) developed linear Gaussian models for handling right-censored data using the technique of data augmentation (Tanner and Wong, 1987). Variation of nonGaussian censored variables can be investigated using survival analysis techniques (Cox, 1972; Prentice and Gloeckler, 1978) because of their ability to properly account for censored and uncensored data information.

Objectives of this study were to propose statistical models for the analysis of coagulation time suitable for making use of coagulating and $\mathrm{NC}$ milk information, to estimate genetic variation and heritability $\left(h^{2}\right)$ of investigated traits and to obtain correlations for sire rankings based on the used models.

Data were from a study aimed to estimate genetic parameters for MCP in Italian Holstein-Friesian cows 
(Cassandro et al., 2008). A total of 1,025 HF cows were sampled once from January to July 2004. Cows were daughters of $54 \mathrm{AI}$ sires and were distributed in 34 herds located in northern Italy. Details on procedures used to collect milk samples and to measure MCP can be found in Cassandro et al. (2008). Milk samples not forming a curd within 31 min were classified as $\mathrm{NC}$ milk. Pedigree information was supplied by the Italian Holstein-Friesian Cattle Breeders Association (ANAFI, Cremona, Italy) and included all known ancestors of sampled cows. Each sampled cow had at least 3 generations of known ancestors.

Of MCP traits, only measures of RCT (min) were of interest for this study. Statistical analysis was performed using linear, threshold, and survival models as described below. Additional analyses, after the rearrangement of the timeframe originally used (31 min) for the observation of RCT, were also performed. In practical cheese making, milk coagulating after 18 min since rennet addition is considered very unsatisfactory. Based on this consideration, all analyses were repeated using $18 \mathrm{~min}$ as a new endpoint to classify milk samples as NC milk. This increased the proportion of NC samples, but, of course, left unchanged the RCT of samples that were classified as coagulated milk. Throughout the paper, SET31 will be used to refer to the original set of data (with an endpoint at $31 \mathrm{~min}$ ) and SET18 to refer to the data obtained after the rearrangement of the timeframe (with an endpoint at $18 \mathrm{~min}$ ). For the standard linear model (SLM), RCT was the response variable and was modeled as a continuous trait for all records with complete information (coagulated milk). Records of NC samples were omitted because of missing information on RCT. This is a common strategy for the analysis of RCT when NC samples are present (Ikonen et al., 1999; Cassandro et al., 2008). The model included the non-genetic effects of herd, DIM class (10 monthly classes from 5 to 305 DIM, 3 bimonthly classes from 306 to 486 DIM, and 1 class for records collected after 486 DIM) and parity (first, second, and third and higher), and the additive polygenic effect of animal (5,847 animals in the pedigree file). Test-day effects were confounded with herd effects because all cows of each herd were sampled once on the same test day.

The threshold model (THM; Gianola, 1982; Sorensen et al., 1995) was applied to the analysis of milk coagulation occurrence (MCO) as a binary outcome. With THM, records of NC samples were used, but continuous variation of RCT for coagulated samples was neglected. The model accounted for the same non-genetic effects included in SLM, but sire effects were used in place of animal genetic effects. Details on model implementation and assumptions on prior distributions for non- genetic and sire effects can be found in Cecchinato et al. (2010).

Models able to handle the censored information of NC milk were a right-censored a linear Gaussian (CLM) and a survival (SUM) model. The CLM was similar to the SLM, but data for NC samples were modeled as right-censored records and included as unknown parameters in the statistical model as described in Sorensen et al. (1998) and Guo et al. (2001). Model implementation and assumptions on prior distributions were as in Sorensen et al. (1998). Effects included in the CLM were the same effects considered in the SLM.

With the SUM, the coagulation function, which is equivalent to the survival function in the context of failure time studies, was preliminary estimated for the general population using the Kaplan-Meier method (Kaplan and Meier, 1958). The assumption of a Weibull distribution for the baseline instantaneous coagulation rate function $\mathrm{cr}_{0}(\mathrm{t})$ was rejected because the relation between $\ln \{-\ln [C(t)]\}$, where $C(t)=$ Kaplan-Meier estimated coagulation function, and the log time was not linear. Hence, the distribution function for $\mathrm{cr}_{0}(\mathrm{t})$ was left completely unspecified and the model became a semiparametric proportional hazard model (Cox, 1972). Effects included in the SUM were the same effects considered in the THM. Prior distributions (multivariate normal and log-gamma distributions for sire and herd effects, respectively) were combined with the likelihood function of the data to obtain an expression proportional to the joint posterior density of all parameters. Estimates of variance components were obtained by Laplacian approximation of the marginal posterior densities. Technical details of the random parameter estimation in survival analysis are given in Ducrocq and Casella (1996). The analysis was carried out using the Survival Kit software (http://www4.jouy.inra.fr/ gabi/les-Ressources/Developpement-d-outils/LogicielKit-de-survie).

Equations in the SLM, CLM, and THM were solved performing numerical integration with the Gibbs sampling technique (Gelfand and Smith, 1990). The analysis was carried out using the program TM (http://snp. toulouse.inra.fr/ alegarra). Improper priors were used for systematic effects and dispersion parameters. Normal priors were assumed for animal additive genetic, sire, herd, and residual effects. Additive relationships between animals (SLM and CLM) or sires (THM and SUM) were taken into account. A single chain of 800,000 Gibbs samples was obtained for each analysis, with a burn-in of 50,000 samples. The length of the burn-in and of the chain were calculated following the methods of Raftery and Lewis (1992) and Geyer (1992), respectively. Gibbs samples were saved at intervals of 
100 iterations. Features of the marginal posterior distributions, convergence analysis, and estimates of Monte Carlo error, were obtained using the Bayesian Output Analysis (BOA) package (http://www.public-heath. uiowa.edu/boa). The posterior median was used as a point estimate of parameters. Lower and upper bounds of the $95 \%$ highest posterior density region for variance components and heritability, and the posterior probability for heritability of being greater than 0.1 were obtained from the estimated marginal densities.

The heritability of RCT in the SLM and CLM analysis was computed as

$$
h^{2}=\frac{\sigma_{A}^{2}}{\sigma_{A}^{2}+\sigma_{H}^{2}+\sigma_{E}^{2}},
$$

where $\sigma_{A}^{2}, \sigma_{H}^{2}$, and $\sigma_{E}^{2}$ are the additive genetic, herd, and residual variance, respectively. Heritability of MCO on the liability scale was computed as

$$
h^{2}=\frac{4 \sigma_{s}^{2}}{1+\sigma_{s}^{2}+\sigma_{H}^{2}},
$$

where $\sigma_{s}^{2}$ and $\sigma_{H}^{2}$ are variance components for sire and herd effects, respectively. With SUM, equivalent heritabilities were computed as in Yazdi et al. (2002).

Spearman correlations for sire rankings based on solutions of models were estimated. Point estimates of sire PTA or EBV were the means of marginal posterior distributions of sire (SUM and THM), or animal (SLM and CLM) effects. The sign of PTA predicted with the SUM was reversed so that positive PTA indicated shorter RCT relative to the population mean and positive correlations were evidence of similar rankings for different models. Pearson correlations between changes in sire rankings (CSR) obtained with the CLM, SUM, and THM, relative to rankings obtained with the SLM, and the percentage of cows yielding NC milk in sire progeny were computed for SET31. The change in sire rankings was computed as CSR $=\operatorname{rank}_{\mathrm{SLM}}-\operatorname{rank}_{\mathrm{x}}$, where rank $_{\mathrm{SLM}}$ was the sire ranking obtained with the SLM and $\operatorname{rank}_{\mathrm{x}}$ was the ranking obtained with the CLM, SUM, or THM.

The average RCT for coagulated samples was 13.9 and $16.9 \mathrm{~min}$ for SET31 and SET18, respectively. As expected, forcing the endpoint for the observation of milk coagulation to 18 min decreased variation of $\mathrm{RCT}$ of coagulated samples. Variation of RCT for SET31 $(\mathrm{SD}=4.7 \mathrm{~min})$ was twice as large as that of samples coagulating within $18 \mathrm{~min}(\mathrm{SD}=2.3 \mathrm{~min})$.
The proportion of censored records in SET31 (i.e., samples not exhibiting coagulation after $31 \mathrm{~min}$ since rennet addition) was $9.7 \%$. When the censoring time was forced to $18 \mathrm{~min}$, the proportion of censored records increased to $44.0 \%$. Noncoagulation of milk is a common problem in many dairy cattle breeds. As reported by Ikonen et al. (1999), noncoagulation of milk is a common problem in the Finnish Ayrshire and some cows can produce NC milk at almost every sampling. Even though several non-genetic effects have been associated with this condition, these effects are only partially responsible for the occurrence of noncoagulation. Ikonen et al. (1999) detected considerable variation in the proportion of NC milk across sire families and observed that many cows producing NC milk were daughters of closely related sires. This suggested a possible genetic cause for noncoagulation of milk.

Features of marginal posterior densities of variance components and heritabilities for the investigated traits are presented in Table 1. Linear models differed in the ability of making use of information provided by $\mathrm{NC}$ samples. As for many other studies investigating variation of RCT (Ikonen et al., 1999; Cecchinato et al., 2009; Vallas et al., 2010), the use of SLM neglected information of NC samples and records of milk that did not coagulate within 31 (SET31) or 18 (SET18) min were not considered. Discarding NC milk records entails loss of the most relevant information when MCP are of interest and it is expected to bias the estimated parameters to an extent that is dependent upon the prevalence of noncoagulation. With the CLM, the Markov chain Monte Carlo procedure generated the unobserved RCT value of $\mathrm{NC}$ milk records by sampling from a truncated normal distribution. Moreover, continuous variation of $\mathrm{RCT}$ for coagulated samples was not neglected as when the THM is used and milk coagulation records are considered after performing a categorization on the binary scale. As a consequence, analyses with the SLM and CLM used different sets of data and comparing their predictive ability was not feasible.

In general, the SLM and CLM estimated larger additive genetic, herd, and residual variance components for SET31 than for SET18 (Table 1). This is attributable to the decreased variation of RCT of coagulated samples in SET18 relative to SET31. Analyses using the CLM gave greater estimates of additive genetic, herd, and residual variances than did the SLM (Table 1). The difference between the SLM and CLM in the estimated variances increased when the proportion of $\mathrm{NC}$ samples in the data increased and can be attributed to the data augmentation algorithm. Similar results have been obtained by González-Recio et al. (2006), who reported 
Table 1. Features of marginal posterior densities of heritability $\left(\mathrm{h}^{2}\right)$, additive genetic $\left(\sigma_{a}^{2}\right)$, herd $\left(\sigma_{h}^{2}\right)$, and residual $\left(\sigma_{e}^{2}\right)$ variance obtained in the analysis of coagulation time (RCT, min) with standard linear (SLM), censored linear (CLM), and survival (SUM) models and of milk coagulation occurrence (MCO) with threshold (THM) models $^{1}$

\begin{tabular}{|c|c|c|c|c|c|c|}
\hline Time $^{2}$ & Parameter & Feature $^{3}$ & \multicolumn{4}{|c|}{ Trait-model $^{4}$} \\
\hline \multirow[t]{7}{*}{ After $31 \mathrm{~min}$} & \multirow[t]{2}{*}{$\sigma_{a}^{2}$} & Median & 4.97 & 9.97 & 0.04 & 0.15 \\
\hline & & LB, UB & $2.10,9.55$ & $4.57,19.94$ & $0,0.18$ & - \\
\hline & \multirow[t]{2}{*}{$\sigma_{h}^{2}$} & Median & 3.41 & 7.28 & 0.30 & 0.22 \\
\hline & & $\mathrm{LB}, \mathrm{UB}$ & $1.86,6.31$ & $4.10,13.63$ & $0.11,0.78$ & - \\
\hline & \multirow[t]{3}{*}{$h^{2}$} & Median & 0.23 & 0.23 & 0.12 & 0.11 \\
\hline & & LB, UB & $0.10,0.42$ & $0.10,0.43$ & $0,0.46$ & - \\
\hline & & $P\left(\mathrm{~h}^{2}>0.1\right)$ & 0.97 & 0.98 & 0.58 & - \\
\hline \multirow[t]{5}{*}{ After $18 \mathrm{~min}$} & \multirow[t]{2}{*}{$\sigma_{a}^{2}$} & Median & 1.10 & 4.55 & 0.04 & 0.24 \\
\hline & & $\mathrm{LB}, \mathrm{UB}$ & $0.31,2.41$ & $2.14,8.56$ & $0,0.11$ & - \\
\hline & $\sigma_{h}^{2}$ & Median & 0.64 & 3.99 & 0.33 & 0.36 \\
\hline & \multirow{2}{*}{$h^{2}$} & $\mathrm{LB}, \mathrm{UB}$ & $0.06,0.41$ & $0.11,0.44$ & $0.02,0.32$ & - \\
\hline & & $P\left(\mathrm{~h}^{2}>0.1\right)$ & 0.90 & 0.98 & 0.61 & - \\
\hline
\end{tabular}

${ }^{1}$ Noncoagulated samples were not considered when coagulation time was analyzed with the SLM.

${ }^{2}$ The time at which assessment of coagulation of individual milk samples ended was 31 or 18 min after rennet addition.

${ }^{3}$ Median is the median of the estimated marginal posterior density of the parameter; LB and UB are the lower and upper bounds of the $95 \%$ highest posterior density region; $P\left(\mathrm{~h}^{2}>0.1\right)$ is the posterior probability for values of $\mathrm{h}^{2}$ greater than 0.1 .

${ }^{4}$ For survival models, $\sigma_{a}^{2}$ was obtained using the mode of the approximated marginal density of $\sigma_{\text {sire }}^{2}$ (the sire variance), $\sigma_{h}^{2}$ was calculated as $\sigma_{h}^{2}=\operatorname{trigamma}(\gamma)$, and $\gamma$ was the parameter of the log-gamma distribution; $h^{2}$ was computed as $h^{2}=\frac{4 \sigma_{\text {sire }}^{2}}{\frac{1}{\bar{p}}+\sigma_{\text {sire }}^{2}+\sigma_{h}^{2}}$, where $\bar{p}$ is the average proportion of uncensored records.

that the CLM provided greater variance and heritability estimates than did the SLM when analyzing days open in Spanish Holstein cattle. Conversely, Urioste et al. (2007) obtained heritabilities for days to calving in beef cattle, which were similar for the SLM and CLM. Although the SLM and CLM differed for the estimated variance components, no (SET31) or slight (SET18) differences were observed between these models for the estimated heritability. Heritability estimates obtained with the SLM were slightly greater for SET31 than for SET18, whereas the CLM provided larger estimates for SET18 than for SET31. The posterior probability of the estimated heritability being greater than 0.1 was larger than $90 \%$ for both models and censoring scenarios.

Threshold model analysis resulted in low estimated heritability and moderate posterior probability of heritability being greater than 0.1 . Estimated parameters obtained with the THM were very similar across censoring scenarios, but $95 \%$ highest posterior density regions were smaller for SET18 than for SET31.

When the censoring time was restricted to $18 \mathrm{~min}$ and the SUM was used, the estimated sire variance increased proportionally more than herd variance, leading to greater heritability estimates than when using SET31 (Table 1). However, due to a greater proportion of censored records in SET18 than SET31, estimates of equivalent heritabilities (Yazdi et al., 2002) were similar across censoring scenarios and comparable to the THM estimates. Estimated parameters obtained with the THM or SUM cannot be compared with those estimated using the SLM and CLM because of the different nature and scale of analyzed traits.

Spearman correlations for sire rankings obtained with the investigated statistical models are reported in Table 2. The estimated correlations indicated that the 4 models gave different sire rankings. Rank correlations of the CLM and SUM with the SLM were greater when the proportion of $\mathrm{NC}$ samples was lower. Correlations between the CLM and SUM were large and greater than those of the CLM or SUM with the THM. These correlations were lower than 0.71 for SET31, but increased when the proportion of NC samples increased (SET18). Within model, correlations between sire rankings obtained for SET31 and SET18 were high for the 
Table 2. Spearman correlations for sire rankings based on different trait-model combinations ${ }^{1,2}$

\begin{tabular}{|c|c|c|c|c|c|c|c|c|}
\hline $\begin{array}{l}\text { Censoring } \\
\text { time }\end{array}$ & Trait-model & \multicolumn{3}{|c|}{ Censoring time at $31 \mathrm{~min}$} & \multicolumn{4}{|c|}{ Censoring time at $18 \mathrm{~min}$} \\
\hline \multirow[t]{3}{*}{$31 \mathrm{~min}$} & RCT-CLM & 0.878 & 0.643 & 0.818 & 0.876 & 0.809 & 0.783 & 0.545 \\
\hline & MCO-THM & & & 0.237 & 0.371 & 0.416 & 0.516 & 0.232 \\
\hline & RCT-SLM & & & & 0.921 & 0.844 & 0.757 & 0.597 \\
\hline $18 \mathrm{~min}$ & RCT-CLM & & & & & 0.887 & 0.770 & 0.711 \\
\hline
\end{tabular}

\footnotetext{
${ }^{1}$ The time at which assessment of coagulation of individual milk samples ended was 31 or 18 min after rennet addition.

${ }^{2}$ RCT-SUM: analysis of rennet coagulation time (RCT) with a survival model (SUM), also considering noncoagulated milk samples; MCOTHM: analysis of milk coagulation occurrence (MCO) with a threshold model (THM); RCT-SLM: analysis of RCT with a standard linear model (SLM), discarding noncoagulated milk samples; RCT-CLM: analysis of RCT time with a censored linear model (CLM), also considering noncoagulated milk samples.
}

CLM and SUM, indicating that rankings provided by these models tended to be stable when a large fraction of samples with observed RCT was reclassified as NC milk, as a consequence of 18-min censoring. For the THM and SLM, the correlations between rankings for SET31 and SET18 were lower than those for the CLM and SUM and ranged from 0.52 to 0.60 . These results indicate that sire rankings obtained with the CLM or SUM were less sensitive to the occurrence of noncoagulation relative to those provided by models neglecting variation of RCT (THM) or discarding information of NC milk (SLM). Despite a large proportion of samples being classified as NC milk, analysis of SET18 with the CLM and, to a lesser extent, with the SUM, gave sire rankings highly correlated with those obtained when the SLM was used to analyze SET31. This result suggests a better behavior of the CLM and SUM relative to the THM and SLM when the data include NC milk information.

Fraction of daughters yielding quickly coagulating milk or NC milk markedly differed across sires. Some sires had a few daughters producing well-coagulating milk, with up to $40 \%$ of the daughters yielding NC milk. Conversely, other sires had a few daughters producing $\mathrm{NC}$, and their daughters' distribution was clearly skewed toward low RCT values (data not shown). To evaluate the effect of incorporating $\mathrm{NC}$ milk information in sire evaluations, we assessed for SET31 the correlation between CSR, the change in sire rankings obtained with the CLM, SUM, and THM, relative to the SLM, and the percentage of $\mathrm{NC}$ milk in sire progeny records. The correlation was $-0.72,-0.65$, and -0.59 for the CLM, SUM, and THM, respectively. These results indicated that including NC milk records in the statistical analysis changes sire rankings compared with the SLM. Being negative, the estimated correlations suggest that increased proportions of daughters yielding $\mathrm{NC}$ milk were associated with a lower sire ranking (i.e., lower genetic merit and negative CSR) relative to the SLM rankings. This result supports the inclusion of NC milk records in genetic evaluation procedures to avoid biased predictions of sire merits for MCP.

Models to incorporate information on NC samples in the analysis of MCP have been proposed. Comparison of predictive ability of models was not feasible due to across-model differences in trait definitions and ability to of make use of different sets of data. In comparison with the CLM and SUM, use of the THR implied a different trait definition, which led to the loss of information provided by continuous variation of RCT of coagulated samples. Future investigations based on bivariate analyses of RCT using the CLM and MCO using the THR might clarify whether these traits are genetically the same or not. Estimated heritabilities and sire rankings based on genetic merit for coagulation ability differed across models. Results of this study indicate that the CLM and SUM are more suitable than the SLM and THR when data contain NC milk information. Although no clear evidence of differences between the CLM and SUM in the ability of exploiting NC milk information has been obtained in this study, other features are of relevance for the choice of the most appropriate model. Animal models are considered to be superior to sire models. Use of animal models with the SUM is still a critical issue, but is not a shortcoming for the application of the CLM. Moreover, extending the application of the CLM to multivariate analysis is straightforward, whereas approximations must be used when performing multiple-trait analyses combining a trait studied using the SUM and other linear traits, especially when nonzero residual correlations between traits exist.

\section{ACKNOWLEDGMENTS}

The authors acknowledge the Italian Ministry of University and Research for financial support (PRIN2003- 
prot.2003078003_002) and the Italian Holstein-Friesian Cattle Breeders Association (ANAFI) for having provided pedigree data. The authors also acknowledge E. López de Maturana [Departamento de Mejora Genética Animal, Instituto Nacional de Investigación y Tecnología Agraria y Alimentaria (INIA), Madrid, Spain] for the use of the TM program, V. Ducrocq [Unité Mixte de Recherche (UMR) 1313 Génétique Animale et Biologie Intégrative (GABI), Institut National de la Recherche Agronomique (INRA), Jouy-en-Josas, France] for the use of the Survival Kit program, and J. Casellas (Departament de Ciència Animal i dels Aliments, Universitat Autònoma de Barcelona, Bellaterra, Spain) for useful comments and suggestions.

\section{REFERENCES}

Cassandro, M., A. Comin, M. Ojala, R. Dal Zotto, M. De Marchi, L. Gallo, P. Carnier, and G. Bittante. 2008. Genetic parameters for milk coagulation properties and their relationships with milk yield and quality traits in Italian Holstein Cows. J. Dairy Sci. 91:371-376.

Cecchinato, A., M. De Marchi, L. Gallo, G. Bittante, and P. Carnier. 2009. Mid-infrared spectroscopy predictions as indicator traits in breeding programs for enhanced coagulation properties of milk. J. Dairy Sci. 92:5304-5313.

Cecchinato, A., O. González-Recio, E. López de Maturana, L. Gallo, and P. Carnier. 2010. A comparison between different survival and threshold models with an application to piglet pre-weaning survival in a dry-cured ham-producing crossbred line. J. Anim. Sci. $88: 1990-1998$.

Cox, D. R. 1972. Regression models and life-tables. J. R. Stat. Soc. [Ser B] 34:187-220.

Ducrocq, V., and G. Casella. 1996. A Bayesian analysis of mixed survival models. Genet. Sel. Evol. 28:505-529.

Gelfand, A., and A. F. M. Smith. 1990. Sampling-based approaches to calculating marginal densities. J. Am. Stat. Assoc. 85:398-409.

Geyer, C. J. 1992. Practical Markov chain Monte Carlo. Stat. Sci. $7: 473-483$.
Gianola, D. 1982. Theory and analysis of threshold characters. J. Anim. Sci. 54:1079-1096.

González-Recio, O., Y. M. Chang, D. Gianola, and K. A. Weigel. 2006. Comparison of models using different censoring scenarios for days open in Spanish Holstein cows. Anim. Sci. 82:233-239.

Guo, S. F., D. Gianola, R. Rekaya, and T. Short. 2001. Bayesian analysis of lifetime performance in Landrace sows using a linear mixed model with censoring. Livest. Prod. Sci. 72:243-252.

Ikonen, T., K. Ahlfors, R. Kempe, M. Ojala, and O. Ruottinen. 1999. Genetic parameters for the milk coagulation properties and prevalence of non-coagulating milk in Finnish dairy cows. J. Dairy Sci. $82: 205-214$

Kaplan, E. L., and P. Meier. 1958. Nonparametric estimation from incomplete observations. J. Am. Stat. Assoc. 53:457-481.

Prentice, R. L., and L. A. Gloeckler. 1978. Regression analysis of grouped survival data with application to breast cancer data. Biometrics 34:57-67.

Raftery, A. E., and S. M. Lewis. 1992. How many iterations in the Gibbs Sampler? Pages 763-774 in Bayesian Statistics IV. J. M. Bernardo, J. O. Berger, A. P. Dawid, and A. F. M. Smith, ed. Oxford Univ. Press, New York, NY.

Sorensen, D., D. Gianola, and I. Korsgaard. 1998. Bayesian mixedeffects model analysis of a censored normal distribution with animal breeding applications. Acta Agric. Scand. Sector A Anim. Sci. 48:222-229.

Sorensen, D. A., S. Andersen, D. Gianola, and I. Korsgaard. 1995. Bayesian inference in threshold models using Gibbs sampling. Genet. Sel. Evol. 27:229-249.

Tanner, M. A., and W. H. Wong. 1987. The calculation of posterior distributions by data augmentation. J. Am. Stat. Assoc. 82:528550

Tyrisevä, A. M., T. Vahlsten, O. Ruottinen, and M. Ojala. 2004. Noncoagulation of milk in Finnish Ayrshire and Holstein-Friesian cows and effect of herds on milk coagulation ability. J. Dairy Sci. 87:3958-3966.

Urioste, J. I., I. Misztal, and J. K. Bertrand. 2007. Fertility traits in spring-calving Aberdeen Angus cattle. 2. Model comparison. J. Anim. Sci. 85:2861-2865.

Vallas, M., H. Bovenhuis, T. Kaart, K. Pärna, H. Kiiman, and E. Pärna. 2010. Genetic parameters for milk coagulation properties in Estonian Holstein cows. J. Dairy Sci. 93:3789-3796.

Yazdi, M. H., P. M. Visscher, V. Ducrocq, and R. Thompson. 2002. Heritability, reliability of genetic evaluations and response to selection in proportional hazard models. J. Dairy Sci. 85:1563-1577. 